\title{
Hyperinflammation and Immune Response Generation in COVID-19
}

\author{
Kamla Prasad Mishra ${ }^{a}$ Ajay Kumar Singh ${ }^{a}$ Shashi Bala Singh ${ }^{b}$ \\ aDefence Research and Development Organization (DRDO)-HQ, New Delhi, India; ${ }^{b}$ National Institute of \\ Pharmaceutical Education and Research (NIPER), Hyderabad, India
}

\section{Keywords}

COVID-19 · SARS-CoV-2 · Inflammation · Immunity · Cytokine

\section{Abstract}

Severe acute respiratory syndrome coronavirus-2 (SARSCoV-2) that causes coronavirus disease 2019 (COVID-19) pandemic has affected millions of people worldwide. The pathophysiology of this virus is not very clearly known, thus, enormous efforts are being made by the scientific community to delineate its evading mechanism. In this review, we have summarized the hyperinflammation and humoral and cell-mediated immune response generated in human body after infection with the SARS-CoV-2 virus. The inflammatory response generated after infection by increased proinflammatory cytokines and chemokines, and complement proteins activation may likely contribute to disease severity. We also discussed the other factors that may affect immunity and could be important comorbidities in the disease severity and outcome.

(c) 2020 S. Karger AG, Basel

karger@karger.com

(c) 2020 S. Karger AG, Basel

www.karger.com/nim

Karger"

\section{Introduction}

The emergence of severe acute respiratory syndrome coronavirus-2 (SARS-CoV-2) from Wuhan, China, that causes COVID-19 poses an unprecedented health crisis. It was declared a pandemic by the WHO on March 11, 2020. Coronaviruses comprise of 4 genus namely alpha coronaviruses, beta coronaviruses, gamma coronaviruses, and delta coronaviruses. SARS-CoV-2 belongs to the beta coronavirus genus. Its closest relative among human coronaviruses is SARS-CoV, with $79 \%$ genetic similarity. However, among all known coronavirus sequences, SARS-CoV-2 is most similar to bat coronavirus RaTG13, with $\sim 96 \%$ similarity, and coronavirus sequences in the pangolin (a scaly anteater mammal) with $\sim 91.02 \%$ similarity [1]. The receptor-binding domain of spike protein of pangolin CoV and SARS-COV-2 has the same 6 amino acids which interact with angiotensin-converting enzyme- 2 receptor while 5 of these 6 residues differ between SARS-CoV-2 and SARS-CoV. However, the polybasic cleavage site of spike protein is different that makes SARS$\mathrm{CoV}-2$ more aggressive for cellular infection in humans [1]. 
Fig. 1. Annotation of SARS-CoV-2 proteins. SARS, severe acute respiratory syndrome; $\mathrm{CoV}$, coronavirus.

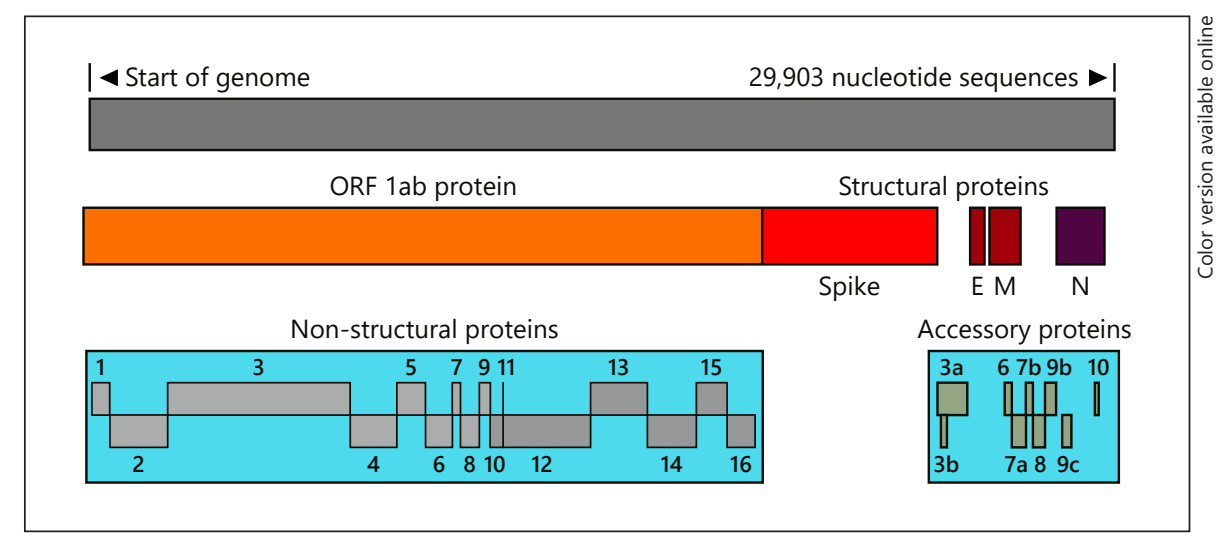

SARS-CoV-2 is + sense, single-strand RNA virus which has about 29,903 nucleotide sequences which makes about 14 genes that makes $\sim 27$ proteins $[1,2]$. It includes nonstructural proteins (nsp 1-16), and nsp 12 is known as RNA-dependent RNA polymerase or RdRp that makes more RNA copy of the virus a target of Remdesivir, a promising drug for COVID-19. Structural proteins include spike $(\mathrm{S})$, envelope $(\mathrm{E})$, membrane protein $(\mathrm{M})$, and nucleocapsid (N) proteins (Fig. 1). The S proteins make crown-like spikes give coronaviruses their name. Others are accessory proteins.

Enormous scientific literatures have been generated during the last 4-5 months on the understanding of immune response as well as immunopathogenesis induced by SARS-CoV-2. Here, we have reviewed the innate and adaptive immunity along with other factors which may contribute to immune modulation in COVID-19 patients.

\section{Differential Immune Response Generation in Mild and Severe COVID-19 Patients}

A schematic diagram of immune response generation in COVID-19 is described in Figure 2. There are many factors which may be responsible for differential immunity in mild and severe COVID-19 cases. Some of them are described as under:

\section{Role of Innate Immunity in Virus Clearance}

The SARS-CoV-2 primarily infects type-II pneumocytes of upper and lower respiratory tract cells. The receptor-binding domain of spike protein interacts with mem- brane-bound angiotensin-converting enzyme- 2 of the host cells and facilitates entry inside host cell [3]. After getting entry inside the cell, virus replicates and infects more cells. Increased number of virus cause the host cell to undergo pyroptosis and release damage-associated molecular patterns (DAMPs), including nucleic acids and ASC oligomers. These DAMPs are recognized by pattern recognition receptors present on lung epithelial cells, endothelial cells, and alveolar macrophages, triggering the generation of proinflammatory cytokines and chemokines. Recognition of viral RNAs by RIG-1, MDA-5, and TLR3 triggers type I interferon production (IFN- $\alpha$ and IFN- $\beta$ ) which protects from any viral infection. In the in vitro studies, Lokugamage et al. [4] and Mantlo et al. [5] demonstrated that SARS-CoV-2 is sensitive to IFN-I pretreatment $[4,5]$. However, the role of interferon stimulating genes still needs to be elucidated in case of SARSCoV-2 infection.

In young individuals, after recognition of viral DAMPs, immediately type I interferon is secreted and virus replication is inhibited [6]. Probably, this could be a reason for the majority of positive cases being asymptomatic. However, in old individuals with comorbid conditions, due to immune senescence, delayed IFN-I production may lead to further recruitment of inflammatory cells such as monocytes, macrophages, and neutrophils. These cells secrete huge proinflammatory cytokines known as cytokine storms that damage the lung alveoli causing severe acute respiratory syndrome. It then turns into sepsis causing dangerously low blood pressure and can damage multiple organ systems [7]. A study conducted by Petrilli et al. [8] in New York on 4,103 patients found early elevations of inflammatory marker C-reactive protein and D-dimer, which had the strongest association with the requirement of ventilator support for patients or death [8]. 


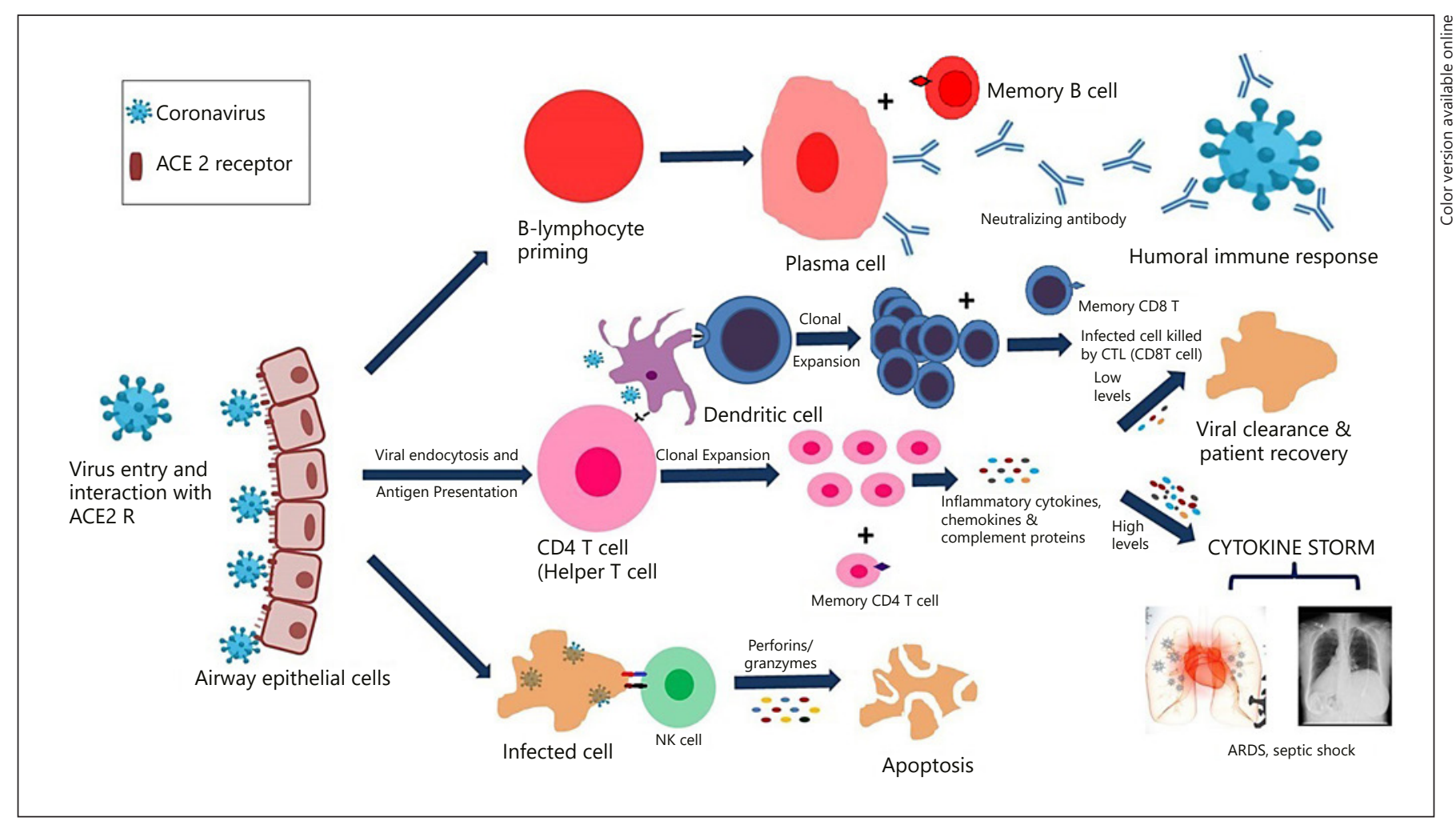

Fig. 2. Immune response generation in COVID-19. COVID-19, coronavirus disease.

\section{Natural Killer Cells}

Natural Killer (NK) cells are large granular lymphocytes which kill virus-infected cells. In severe COVID-19 patients, a significantly reduced number of NK cells were reported in the peripheral blood mononuclear cells as compared to age-matched nonsevere COVID-19 patients [9-12]. The NK cells are nonresident lung cells; however, they can infiltrate into lungs from peripheral blood via its chemokine receptor CXCR3 towards chemoattractant ligands secreted by monocytes and macrophages in the lungs of COVID-19 patients [13,14]. Wilk et al. [15] have reported the reduced intracellular granzyme B level in NK cells indicating that NK cell cytotoxicity is reduced in severe COVID-19 patients.

\section{Complements Activation and Hyperinflammation}

The complement system is part of the innate immune response and is involved in the initiation of proinflammatory responses that protects from bacterial infections, and often neutralizes invading viruses. It has 3 pathways that converge on the common pathway[16]. The activation of the classical pathway occurs by immune complexes, the alternative pathway triggered by specific surface antigens and lectin pathway induced by binding mannose residues on the pathogen surface. The common pathway includes production of $\mathrm{C} 3 \mathrm{a}$ and $\mathrm{C} 5 \mathrm{a}$ which triggers inflammatory response. Clinical data on the role of complement activation in the development of SARS-CoV-2-associated ARDS are very limited. A recent study reported that lung biopsy samples from patients with severe COVID-19 showed activation of complement lectin pathway, showing aberrant $\mathrm{C} 3 \mathrm{a}$ generation and $\mathrm{C} 3$-fragment deposition. A significant increase of serum C5a levels was also observed. Importantly, treatment of patients with an antiC5a antibody led to immediate clinical improvement, as measured by increased lung oxygenation and decreased systemic inflammation [17]. Some of the severe COVID-19 patients showed thromboembolic disorder this may be attributed to thrombo-inflammatory processes $[18,19]$. Autopsy reports of SARS patients had also shown coagulopathy without the infiltrates of virus that can occur in many different clinical scenarios including pathogenic complement activation [20]. The unrestrained acti- 
vation of complement pathway induced by the SARSCoV-2 plays a major role in acute and chronic inflammation, endothelial cell dysfunction, thrombus formation, and intravascular coagulation, and ultimately contributes to multiple organ failure and death. Risitano et al. [21] reported that activation of complement cascade may lead to maladaptive inflammatory response; hence, inhibition of complement C3 and C5 may have therapeutic potential on SARS-CoV-2 induced hyperinflammation in severe COVID-19 patients.

\section{Adaptive Immunity and T- and B-Cell Response}

The adaptive immune response involves $\mathrm{T}$ and $\mathrm{B}$ lymphocytes. The cell-mediated immunity is driven by T lymphocytes while humoral immunity is driven by $\mathrm{B}$ lymphocytes. When viruses infect the cell, the viral peptides are loaded on MHC class I of nucleated cells such as epithelial cells, monocytes, dendritic cells, etc. These viral peptides are recognized by cytotoxic $\mathrm{T}$ lymphocytes (CD8 T cells), and infected cells are subsequently killed by cytotoxic T lymphocytes by apoptosis. The secreted proteins of viruses are also loaded on class II MHC molecules and interact with T-cell receptor of CD4 T cells (helper T cells). CD4 T cells secrete cytokines like IL-2 and IL- 6 which help virus-specific B cell to proliferate. B cells become secretory cells called plasma cells, and memory and plasma cells secrete IgM, IgG, and IgA antibodies specifically to neutralize the viruses [16]. These processes take a little time to develop. In case of COVID-19 published literature suggested that acute antibody responses to SARS-CoV-2 generate within 19 days after symptom onset [22]. Seroconversion for IgG and IgM occurred simultaneously or sequentially [22].

Ju et al. [23] reported that some degree of cross-reactivity to SARS-CoV-1 S and N proteins and to MERSCoV S protein was found in the plasma from COVID-19 patients, but, no cross-reactivity was found to the RBD from SARS-CoV-1 and MERS-CoV. In addition, plasma from COVID-19 patients did not neutralize SARS-CoV-1 or MERS-CoV [23], thus, SARS-CoV-2 and SARS-CoV are immunologically distinct. Memory B-cell response is important to prevent further infection of SARS-CoV-2. A study conducted by Bao et al. [24] in rhesus macaques indicated that macaques that had resolved the primary infection developed immunity against SARS-CoV-2 and 28 days after rechallenge no symptoms of COVID-19 appear [24]. This study gives a clue that protective immu-

Immunity in COVID-19 Patients nity could be generated from vaccines being tested for COVID-19. However, the long-term memory response is yet to be studied because of the timing.

\section{CD4 and CD8 T Cells in COVID-19}

Several studies have reported the reduced lymphocyte counts with significantly reduced number of CD4 and CD8 T cells in COVID-19 patients [24-27]. In severe COVID-19 cases, the CD8 T cells were drastically reduced $[24,26,27]$; however, in mild cases, the patients had normal or slightly higher CD4 and CD8 T cell counts [28, 29]. Current data point to potentially diverse patterns of $\mathrm{CD} 8+\mathrm{T}$ cell responses in patients with COVID-19. There are few reports which suggest that SARS-CoV-2-specific CD8 $\mathrm{T}$ cells in patients, who have recovered, provide evidence of CD8 T-cell memory in many convalescent patients $[30,31]$.

Zheng et al. [12] reported that expression of NKG2A was increased in cytotoxic T cells and NK cells but the level of granzyme b production was reduced. Also, the percentage of NKG2A + cytotoxic lymphocytes was decreased in recovered patients infected with SARS-CoV-2, signifying that NKG2A expression may be correlated with functional exhaustion of cytotoxic lymphocytes and disease progression in the early stage of COVID-19 [12]. The reports published so far indicating that COVID-19 is associated with both functional exhaustion as well as reduction of T cells.

There is a limited study to clear the actual CD4 and CD8 T-cell response, central, and effector memory T-cell generation against SARS-CoV-2, and more studies are required to clear the exact $\mathrm{T}$ cell response in COVID-19 patients. The virus-specific CD8 T-cell response is important to understand the cell-mediated destruction of SARS-CoV-2 which is tricky to measure.

\section{Other Factors That May Affect Immunity and Severity of Disease}

\section{Age}

It is well established that immunity of young and old individuals differs. Therefore, age may be one of the risk factors. Older people and people with chronic illness are at greater risk. According to the data from China, the older COVID-19 patients are more likely at risk as compared to the younger patients [32]. The recent analysis of Chinese data reported the chance of death in confirmed CO- 
VID-19 cases were more than $13 \%$ for patients above 80 years and older, compared to about $0.15 \%$ for patients in their $30 \mathrm{~s}$, and virtually $0 \%$ for patients under 20 [32].

\section{Ethnicity}

The effects of ethnicity on COVID-19 is yet to be established, however, emerging data from the USA, suggest that death rates among Black/African American persons (92.3 deaths per 100,000 population), and Hispanic/Latino persons (74.3) were substantially higher than that of white (45.2) or Asian (34.5) persons [33]. In a preprint study from the UK, no significant effect of ethnicity itself was found on severe outcomes of COVID-19 patients with adjustment for age/sex/comorbidities [34]. The higher observed incidence and severity in some ethnic groups may be associated with socioeconomic, cultural, or lifestyle factors, genetic predisposition, or pathophysiological differences in susceptibility or response to infection [35].

\section{Comorbidities}

Emerging data on COVID-19 patients who develop serious or fatal COVID-19 are disproportionately likely to have at least 1 major underlying health condition, such as hypertension, obesity, diabetes, asthma, kidney disease, chronic obstructive pulmonary disorder, or chronic inflammatory diseases [8]. Obesity is a main risk factor for these comorbidities and more generally for impaired metabolic health and is also linked to an increased risk of pneumonia [36]. Measurement of anthropometric characteristics such as BMI and metabolic parameters is crucial to better estimate the risk of complications in patients with COVID-19. Obesity represents a risk factor that also puts younger people at increased risk. On the other hand, obesity is a modifiable risk parameter. Nevertheless, in most cases, a large amount of weight loss is difficult to achieve. In this respect, it would be important to mention that achieving metabolic health in obesity is expected not only to reduce the risk of cardiometabolic diseases but also of a severe course of COVID-19 [37].

\section{Diet}

Eating right is equally important to enhance immunity. Therefore, balanced diet is always advised. The diet rich in high fiber content is helpful as it strengthens gut microbes which strengthen immunity and sleep well as it is proven immune booster. Vitamin $\mathrm{D}$ also helps in boosting immunity. It is produced by the body in response to sunlight and has major health benefits. Deficiency in vitamin D may pose at greater risk to infectious diseases. A majority of global population is vitamin D deficient. Therefore, food with rich in vitamin $\mathrm{D}$ is required to fulfill the daily need [38-40]. Yoga and moderate exercise are also helpful in strengthening immunity [41], so regular practice of yoga and moderate exercise will strengthen immunity. One should not be panic or stressed because stress lowers immunity. The strong immune system is able to kill the virus at the early stage of infection.

\section{Herd Immunity}

If the majority of the population is immune to an infectious agent, the chance of a susceptible individual contacting an infecting individual is so low that the susceptible individual is not likely to become infected. This is known as herd immunity [16, 42-45]. The possible ways to build widespread SARS-CoV-2 immunity are (a) natural immunization of global population with the virus over time and (b) vaccination of global population with an effective and safe vaccine. The consequences of former approach could be far reaching as millions of people may die due to natural infection of virus and a sudden boom in sick people needing ICU care will overwhelm hospitals. Slowing it down by imposing restrictions would mean health systems could be spared and lives saved. Therefore, in the absence of a vaccine, establishing herd immunity should not be the ultimate goal.

\section{Conclusion}

The rapid spread of SARS-CoV-2 from Wuhan, China, to all 6 continents in a very short span of time has posed urgency in both basic science and clinical research, and looking at the tremendous amounts of information generated every day is remarkable by the scientific community. A significant knowledge has been generated in a short time about the understanding of immunology of SARS-CoV-2 infection. The epidemiological data emerging from world suggest higher transmissibility rate of SARS-CoV-2 as compared to the previous coronaviruses such as SARS and MERS. Here, we have reviewed the innate immunity including NK cell functionality, complement activation, and interferon production in COVID-19 patients and further highlighted the adaptive immune response in terms of humoral and cell-mediated immunity. However, more studies are required to address the differential immune response generation in severe and mild COVID-19 patients. The outcome of studies being conducted on SARS-CoV-2 worldwide will likely provide a robust framework to fulfill that unmet need.
Mishra/Singh/Singh 


\section{Conflict of Interest Statement}

The authors have no conflicts of interest to declare.

\section{Author Contributions}

K.P.M. has written the manuscript, and A.K.S. and S.B.S. have edited and finalized the manuscript.

\section{Funding Sources}

The authors received no financial support.

\section{References}

1 Andersen KG, Rambaut A, Lipkin WI, Holmes EC, Garry RF. The proximal origin of SARS-CoV-2. Nat Med. 2020 Apr;26(4):4502 .

2 Wu F, Zhao S, Yu B, Chen YM, Wang W, Song ZG, et al. A new coronavirus associated with human respiratory disease in China. $\mathrm{Na}$ ture. 2020 Mar;579(7798):265-9.

3 Yan R, Zhang Y, Li Y, Xia L, Guo Y, Zhou Q. Structural basis for the recognition of SARSCoV-2 by full-length human ACE2. Science. 2020 Mar 27;367(6485):1444-8.

4 Lokugamage KG, Hage A, Schindewolf C, Rajsbaum R, Menachery VD. SARS-CoV-2 sensitive to type I interferon pretreatment. BioRxiv. 2020.

5 Mantlo E, Bukreyeva N, Maruyama J, Paessler S, Huang C. Antiviral activities of type I interferons to SARS-CoV-2 infection. Antiviral Res. 2020 Apr 29;179:104811.

6 Hajishengallis G. Too old to fight? Aging and its toll on innate immunity. Mol Oral Microbiol. 2010 Feb;25(1):25-37.

7 Tay MZ, Poh CM, Rénia L, MacAry PA, Ng LFP. The trinity of COVID-19: immunity, inflammation and intervention. Nat Rev Immunol. 2020 Jun;20(6):363-37.

8 Petrilli CM, Jones SA, Yang J, Rajagopalan H, O'Donnell L, Chernyak Y, et al. Factors associated with hospitalization and critical illness among 4,103 patients with Covid-19 disease in New York City. medRxiv. 2020.

9 Song CY, Xu J, He JQ, Lu YQ. COVID-19 early warning score: a multi-parameter screening tool to identify highly suspected patients. MedRxiv. 2020 Jan 1.

10 Wang F, Nie J, Wang H, Zhao Q, Xiong Y, Deng L, et al. Characteristics of peripheral lymphocyte subset alteration in COVID-19 pneumonia. J Infect Dis. 2020 May 11; 221(11):1762-9.

11 Yu L, Tong Y, Shen G, Fu A, Lai Y, Zhou X, et al. Immunodepletion with hypoxemia: a potential high risk subtype of coronavirus disease 2019. medRxiv. 2020 Jan 1.

12 Zheng M, Gao Y, Wang G, Song G, Liu S, Sun $D$, et al. Functional exhaustion of antiviral lymphocytes in COVID-19 patients. Cell Mol Immunol. 2020 May;17(5):533-5.

13 Chu H, Chan JF, Wang Y, Yuen TT, Chai Y, Hou Y, et al. Comparative replication and immune activation profiles of SARS-CoV-2 and SARS-CoV in human lungs: an ex vivo study with implications for the pathogenesis of COVID-19. Clin Infect Dis. 2020 Sep 12;71(6): 1400-9.

14 Liao M, Liu Y, Yuan J, Wen Y, Xu G, Zhao J, et al. The landscape of lung bronchoalveolar immune cells in COVID-19 revealed by single-cell RNA sequencing. medRxiv. 2020 Jan 1 .

15 Wilk AJ, Rustagi A, Zhao NQ, Roque J, Martinez-Colon GJ, McKechnie JL, et al. A singlecell atlas of the peripheral immune response to severe COVID-19. medRxiv. 2020 Jan 1.

16 Kuby J. Kuby immunology. New York: W.H. Freeman; 2013

17 Gao T, Hu M, Zhang X, Li H, Zhu L, Liu H, et al. Highly pathogenic coronavirus $\mathrm{N}$ protein aggravates lung injury by MASP-2-mediated complement over-activation. medRxiv. 2020 Jan 1.

18 Grillet F, Behr J, Calame P, Aubry S, Delabrousse E. Acute pulmonary embolism associated with COVID-19 pneumonia detected by pulmonary CT angiography. Radiology. 2020 Sep;296(3):E186-8.

19 Rotzinger DC, Beigelman-Aubry C, von Garnier C, Qanadli SD. Pulmonary embolism in patients with COVID-19: time to change the paradigm of computed tomography. Thromb Res. 2020 Apr 11;190:58-9.

20 Campbell CM, Kahwash R. Will complement inhibition be the new target in treating COVID-19 related systemic thrombosis? Circulation. 2020 Jun 2;141(22):1739-41.

21 Risitano AM, Mastellos DC, Huber-Lang M, Yancopoulou D, Garlanda C, Ciceri F, et al. Complement as a target in COVID-19? Nat Rev Immunol. 2020 Jun;20(6):343-4.

22 Long QX, Liu BZ, Deng HJ, Wu GC, Deng K, Chen YK, et al. Antibody responses to SARSCoV-2 in patients with COVID-19. Nat Med. 2020 Apr 29.

23 Ju B, Zhang Q, Ge X, Wang R, Yu J, Shan S, et al. Potent human neutralizing antibodies elicited by SARS-CoV-2 infection. BioRxiv. 2020 Mar 21:990770.

24 Bao L, Deng W, Gao, Xiao C, Liu J, Xue J, et al. Lack of reinfection in rhesus macaques infected with SARS-CoV-2. bioRxiv. 2020 May 01.

25 Nie S, Zhao X, Zhao K, Zhang Z, Zhang Z, Zhang Z. Metabolic disturbances and inflammatory dysfunction predict severity of coronavirus disease 2019 (COVID-19): a retro- spective study. medRxiv. 2020 Mar 24: 20042283.

26 Wang W, He J, Wu S. The definition and risks of cytokine release syndrome-like in $11 \mathrm{COV}$ ID-19-infected pneumonia critically ill patients: disease characteristics and retrospective analysis. Medrxiv. 2020 Jan 1.

27 Zheng HY, Zhang M, Yang CX, Zhang N, Wang XC, Yang XP, et al. Elevated exhaustion levels and reduced functional diversity of $\mathrm{T}$ cells in peripheral blood may predict severe progression in COVID-19 patients. Cell Mol Immunol. 2020 May;17(5):541-3.

28 Liu B, Han J, Cheng X, Yu L, Zhang L, Wang $\mathrm{W}$, et al. Persistent SARS-CoV-2 presence is companied with defects in adaptive immune system in non-severe COVID-19 patients. medRxiv. 2020

29 Thevarajan I, Nguyen THO, Koutsakos M, Druce J, Caly L, van de Sandt CE, et al. Breadth of concomitant immune responses prior to patient recovery: a case report of non-severe COVID-19. Nat Med. 2020 Apr; 26(4):453-5.

30 Duan K, Liu B, Li C, Zhang H, Yu T, Qu J, et al. Effectiveness of convalescent plasma therapy in severe COVID-19 patients. Proc Natl Acad Sci U S A. 2020 Apr 28;117(17):9490-6.

31 Ni L, Ye F, Cheng ML, Feng Y, Deng YQ, Zhao H, et al. Detection of SARS-CoV-2-specific humoral and cellular immunity in COVID-19 convalescent individuals. Immunity. 2020 Jun 16;52(6):971-e3.

32 Verity R, Okell LC, Dorigatti I, Winskill P, Whittaker C, Imai N, et al. Estimates of the severity of coronavirus disease 2019: a modelbased analysis. Lancet Infect Dis. 2020 Jun; 20(6):669-77.

33 https: //www.cdc.gov/coronavirus/2019ncov/need-extra-precautions/racial-ethnicminorities.html.

34 Teo JT, Bean D, Bendeyan R, Dobson R, Shah A. Impact of ethnicity on outcome of severe COVID-19 infection. Data from an ethnically diverse UK tertiary centre. medRxiv. 2020 May 2:2007864.

35 Khunti K, Singh AK, Pareek M, Hanif W. Is ethnicity linked to incidence or outcomes of covid-19? BMJ. 2020 Apr 20;369:m1548.

36 Stefan N, Birkenfeld AL, Schulze MB, Ludwig DS. Obesity and impaired metabolic health in patients with COVID-19. Nat Rev Endocrinol. 2020 Apr 23. 
37 Stefan N, Häring HU, Schulze MB. Metabolically healthy obesity: the low-hanging fruit in obesity treatment? Lancet Diabetes Endocrinol. 2018 Mar;6(3):249-58.

38 Aparna P, Muthathal S, Nongkynrih B, Gupta SK. Vitamin D deficiency in India. J Family Med Prim Care. 2018 Mar-Apr;7(2):324-30.

39 Palacios C, Gonzalez L. Is vitamin D deficiency a major global public health problem? J Steroid Biochem Mol Biol. 2014 Oct;144 Pt A: $138-45$.

40 Saul L, Mair I, Ivens A, Brown P, Samuel K, Campbell JDM, et al. 1,25-Dihydroxyvitamin
$\mathrm{D}(3)$ restrains $\mathrm{CD} 4(+) \mathrm{T}$ cell priming ability of $\mathrm{CD} 11 \mathrm{c}(+)$ dendritic cells by upregulating expression of CD31. Front Immunol. 2019 Mar 28;10:600.

41 Venkatesh HN, Ravish H, Wilma Delphine Silvia CR, Srinivas H. Molecular signature of the immune response to yoga therapy in stress-related chronic disease conditions: an insight. Int J Yoga. 2020 Jan 1;13(1):9.

42 Chen J, Ye L, Zhou MY, Cheng YR, Wang MW, Feng ZH. Herd immunity and COVID-19. Eur Rev Med Pharmacol Sci. 2020 Apr;24(8):4064-5.
43 Danchin A, Timmis K. SARS-CoV-2 variants: relevance for symptom granularity, epidemiology, immunity (herd, vaccines), virus origin and containment? Environ Microbiol. 2020 Jun;22(6):2001-6.

44 Syal K. COVID-19: herd immunity and convalescent plasma transfer therapy. J Med Virol. 2020 Sep;92(9):1380-2.

45 Kwok KO, Lai F, Wei WI, Wong SYS, Tang JWT. Herd immunity: estimating the level required to halt the COVID-19 epidemics in affected countries. J Infect. 2020 Jun;80(6):e323. 